\title{
DENSITY OF A MINIMAL SUBMANIFOLD AND TOTAL CURVATURE OF ITS BOUNDARY
}

\author{
Jaigyoung Choe* and Robert Gulliver
}

\begin{abstract}
Given a piecewise smooth submanifold $\Gamma^{n-1} \subset \mathbb{R}^{m}$ and $p \in \mathbb{R}^{m}$, we define the vision angle $\Pi_{p}(\Gamma)$ to be the $(n-1)$-dimensional volume of the radial projection of $\Gamma$ to the unit sphere centered at $p$. If $p$ is a point on a stationary $n$-rectifiable set $\Sigma \subset \mathbb{R}^{m}$ with boundary $\Gamma$, then we show the density of $\Sigma$ at $p$ is $\leq$ the density at its vertex $p$ of the cone over $\Gamma$. It follows that if $\Pi_{p}(\Gamma)$ is less than twice the volume of $S^{n-1}$, for all $p \in \Gamma$, then $\Sigma$ is an embedded submanifold. As a consequence, we prove that given two $n$-planes $R_{1}^{n}, R_{2}^{n}$ in $\mathbb{R}^{m}$ and two compact convex hypersurfaces $\Gamma_{i}$ of $R_{i}^{n}, i=1,2$, a nonflat minimal submanifold spanned by $\Gamma:=\Gamma_{1} \cup \Gamma_{2}$ is embedded.
\end{abstract}

\section{Introduction}

Fenchel [F1] showed that the total curvature of a closed space curve $\gamma \subset \mathbb{R}^{m}$ is at least $2 \pi$, and it equals $2 \pi$ if and only if $\gamma$ is a plane convex curve. Fáry [Fa] and Milnor [M] independently proved that a simple knotted regular curve has total curvature larger than $4 \pi$. These two results indicate that a Jordan curve which is curved at most double the minimum is isotopically simple. But in fact minimal surfaces spanning such Jordan curves must be simple as well. Indeed, Nitsche $[\mathrm{N}]$ showed that an analytic Jordan curve in $\mathbb{R}^{3}$ with total curvature at most $4 \pi$ bounds exactly one minimal disk. Moreover, Ekholm, White and Wienholtz $[E W W]$ proved that a minimal surface spanning such a Jordan curve in $\mathbb{R}^{m}$ is embedded.

Given an $n$-dimensional submanifold $M$ of $\mathbb{R}^{m}$, there are two well-studied ways of defining the total curvature of $M$ : the higher-dimensional Gauss-Bonnet integral $\int_{M} \Omega$ as defined in [AW] and [C1]; and the total absolute curvature of $M, \int_{M} K^{*} d V_{M}$ as defined by Chern and Lashof in [CL] (see section 2 below). Chern and Lashof proved that $\int_{M} K^{*} d V_{M} \geq 2$, with equality if and only if $M$ is a convex hypersurface in an $(n+1)$-dimensional plane. Eells and Kuiper have shown that if $\int_{M} K^{*} d V_{M}<3$ then $M$ is homeomorphic to $\mathbb{S}^{n}$ and that if $\int_{M} K^{*} d V_{M}<4$ then $M$ is homeomorphic to $\mathbb{S}^{n}, \mathbb{R} P^{n}, \mathbb{C} P^{n / 2}, \mathbb{H} P^{n / 4}$ or to Cay $P^{2}$ (for $n=16)$. [EK].

In the light of Ekholm-White-Wienholtz's theorem, it is quite natural to conjecture that an $n$-dimensional minimal submanifold $\Sigma \subset \mathbb{R}^{m}$ spanning a compact connected submanifold $\Gamma^{n-1}$ with total absolute curvature $<4$ is embedded. In this paper we prove a theorem in the spirit of this conjecture: given two $n$-planes $R_{1}^{n}, R_{2}^{n}$ in $\mathbb{R}^{m}$ and two compact convex

\footnotetext{
*The first-named author was supported in part by KRF-2007-313-C00057.
} 
hypersurfaces $\Gamma_{i}^{n-1}$ of $R_{i}^{n}, i=1,2$, a nonflat minimal submanifold spanned by $\Gamma:=\Gamma_{1} \cup \Gamma_{2}$ is embedded.

In $[\mathrm{Fa}]$ Fáry showed that the total curvature of a space curve $\gamma$ in $\mathbb{R}^{m}$ is equal to the average over all 2-planes $R^{2} \subset \mathbb{R}^{m}$ of the total curvature of the orthogonal projection of $\gamma$ onto the $R^{2}$. We shall use an extension of Fáry's theorem, due to Langevin and Shifrin [LS], which shows that given an $(n-1)$-dimensional submanifold $\Gamma$ of $\mathbb{R}^{m}$, the total absolute curvature of $\Gamma$ equals the average over all $n$-planes $R^{n} \subset \mathbb{R}^{m}$ of the total absolute curvature of the orthogonal projection of $\Gamma$ onto the $n$-plane $R^{n}$.

\section{Total absolute curvature}

Consider a submanifold $M^{n}$ of Euclidean space $\mathbb{R}^{m}$. As discussed above, in high dimension and codimension we discuss two types of total curvature: one intrinsic (Allendörfer-WeilChern-Gauss-Bonnet), and one extrinsic (Chern-Lashof). In this section we shall review Chern-Lashof's total absolute curvature. This total curvature may be understood in terms of Gauss-Kronecker curvature of hypersurfaces.

Let $M^{n}$ be an oriented hypersurface immersed in $\mathbb{R}^{n+1}$. A unit normal vector $\nu$ to $M$ at $p \in M$ defines the Gauss map $G_{1}: M \rightarrow \mathbb{S}^{n}$. The determinant of the differential $G_{1} *$, or of the second fundamental form of $M$, is called the Gauss-Kronecker curvature of $M$, which we shall denote $G K_{M}$. It follows that for $M$ compact,

$$
\int_{M} G K_{M} d V_{M}=c_{n} \operatorname{deg}\left(G_{1}\right), \quad c_{n}:=\operatorname{Vol}\left(\mathbb{S}^{n}\right) .
$$

Furthermore, if $n$ is even, H. Hopf $[\mathrm{H}]$ showed

$$
\int_{M} G K_{M} d V_{M}=\frac{1}{2} c_{n} \chi(M) .
$$

Now let $M$ be an $n$-dimensional submanifold of $\mathbb{R}^{m}$. The volume form of the unit normal bundle $N_{1} M$ of $M$ is $d V_{M} \wedge d \sigma_{m-n-1}$ where the restriction of $d \sigma_{m-n-1}$ to a fiber of $N_{1} M$ at $p$ is the volume form of the sphere of unit normal vectors at $p \in M$. Define the Gauss map $G_{1}: N_{1} M \rightarrow \mathbb{S}^{m-1}$ by $G_{1}(p, \nu)=\nu$ and let $d \sigma_{m-1}$ be the volume form of $\mathbb{S}^{m-1}$. Then the Lipschitz-Killing curvature $G(p, \nu)$ of $M$ at $(p, \nu)$ is defined to be the scalar $G(p, \nu)$ such that

$$
G_{1}^{*}\left(d \sigma_{m-1}\right)=G(p, \nu) d V_{M} \wedge d \sigma_{m-n-1} .
$$

Then $G(p, \nu)$ is exactly the volume expansion ratio of $G_{1}$, that is,

$$
G(p, \nu)=\lim _{D \rightarrow\{p\}} \frac{\operatorname{Vol}\left(G_{1}(D)\right)}{\operatorname{Vol}(D)},
$$

where $\operatorname{Vol}\left(G_{1}(D)\right)$ denotes the signed volume of $G_{1}(D)$. In fact, $G(p, \nu)$ has the following geometric interpretation [CL]: $G(p, \nu)$ is equal to the Gauss-Kronecker curvature at $p$ of the orthogonal projection of $M$ onto the $(n+1)$-dimensional plane $L(\nu)$ spanned by $T_{p} M$ and $\nu$.

Let $\pi$ be the canonical projection of $N_{1} M$ into $M$. The integrals

$$
K(p):=\frac{1}{c_{m-1}} \int_{\pi^{-1}(p)} G(p, \nu) d \sigma_{m-n-1} \quad \text { and } \quad K^{*}(p):=\frac{1}{c_{m-1}} \int_{\pi^{-1}(p)}|G(p, \nu)| d \sigma_{m-n-1}
$$


are called the total curvature and the total absolute curvature of $M$ at $p$, respectively. The integrals

$$
\tau(M):=\int_{M} K d V_{M}, \quad \text { and } \quad \tau^{*}(M):=\int_{M} K^{*} d V_{M}
$$

are called the total curvature and the total absolute curvature of $M$, respectively. Lipschitz and Killing have shown that $K(p)$ is an intrinsic quantity of $M$ at $p$ for $n$ even (see [SS] for a more general result). However, $K(p)=0$ for $n$ odd. Both $\tau(M)$ and $\tau^{*}(M)$ remain unchanged even if the ambient space $\mathbb{R}^{m}$ is embedded into $\mathbb{R}^{k}, k>m$.

For $M^{n} \subset \mathbb{R}^{m}$, Fenchel [F2] generalized Hopf's theorem (1):

$$
\int_{M} K d V_{M}=\chi(M)
$$

In contrast, Chern and Lashof [CL] proved that

$$
\int_{M} K^{*} d V_{M} \geq 2
$$

with equality if and only if $M$ is a convex hypersurface in an $(n+1)$-dimensional plane, and that if $\int_{M} K^{*} d V_{M}<3$ then $M$ is homeomorphic to $\mathbb{S}^{n}$. Moreover, Morse theory tells us that

$$
\int_{M} K^{*} d V_{M} \geq \sum_{i} \beta_{i}
$$

where $\beta_{i}$ is the $i$-th Betti number of $M$ ([W], Theorem 28).

\section{$3 \quad$ Vision angle versus average density}

A minimal submanifold $\Sigma^{n}$ in $\mathbb{R}^{m}$ has the remarkable property that the density of $\Sigma$ at $p \in \Sigma$ is bounded above by that of the cone $C=p \circledast \partial \Sigma$ at its vertex $p$. (We assume that $\Sigma$ with its boundary is compact.) Recall that the density of $\Sigma$ is defined as

$$
\Theta_{\Sigma}(p)=\lim _{r \rightarrow 0} \frac{\operatorname{Vol}\left(\Sigma \cap B_{r}^{m}(p)\right)}{\operatorname{Vol}\left(B_{r}^{n}(p)\right)} .
$$

Further, the density of a cone $C$ has the interesting property that it equals the average of the densities of the orthogonal projections of $C$ onto $n$-planes in $\mathbb{R}^{m}$. These properties will be verified in this section.

In what follows, we shall write $\bar{\nabla}$ for the Euclidean connection on $\mathbb{R}^{m}$, and $\nabla=\nabla_{M}$ for the induced connection on a submanifold $M$.

Lemma 1. Let $\Sigma$ be an n-dimensional minimal submanifold of $\mathbb{R}^{m}, p$ a point of $\mathbb{R}^{m}$, and $C$ an $n$-dimensional piecewise smooth cone with vertex $p$. Define the Euclidean distance function $r(x)=\operatorname{dist}(p, x), x \in \mathbb{R}^{m}$. Let $Y_{1}=r \bar{\nabla} r$ and $Y_{2}=r^{1-n} \bar{\nabla} r$, and define $\operatorname{div}_{\Sigma} Y_{i}=\operatorname{tr}_{\Sigma} \bar{\nabla} Y_{i}=$ $\sum_{j}\left\langle\bar{\nabla}_{e_{j}} Y_{i}, e_{j}\right\rangle,\left\{e_{1}, \ldots, e_{n}\right\}$ being an orthonormal frame of $\Sigma$. Then

(a) $O n \Sigma, \operatorname{div}_{\Sigma} Y_{1}=n$ and $\operatorname{div}_{\Sigma} Y_{2} \geq 0$; 
(b) $O n C, \operatorname{div}_{C} Y_{1}=n$ and $\operatorname{div}_{C} Y_{2}=0$.

We require that $C$ be piecewise smooth, that is, a topological manifold which has a triangulation into simplices that are $C^{2}$ up to their boundaries.

Proof. Given an $n$-dimensional submanifold $M \subset \mathbb{R}^{m}$, it is well known that

$$
\triangle_{M} x:=\left(\triangle_{M} x_{1}, \ldots, \triangle_{M} x_{m}\right)=\vec{H}
$$

where $\vec{H}$ is the mean curvature vector of $M$, the trace of its second fundamental form. Hence the orthogonal coordinate functions $x_{1}, \ldots, x_{m}$ of $\mathbb{R}^{m}$ are harmonic on a minimal submanifold $\Sigma^{n}$ of $\mathbb{R}^{m}$. If we take $p$ as the origin, then since $\vec{H}=0$ on $\Sigma$,

$$
\operatorname{div}_{\Sigma}\left(Y_{1}\right)=\operatorname{div}_{\Sigma}(r \bar{\nabla} r)=\frac{1}{2} \triangle_{\Sigma} r^{2}+\langle r \bar{\nabla} r, \vec{H}\rangle=\frac{1}{2} \sum \triangle_{\Sigma} x_{i}^{2}=\sum x_{i} \triangle_{\Sigma} x_{i}+\sum\left|\nabla x_{i}\right|^{2}=n .
$$

On the cone $C$, since $\vec{H}$ is perpendicular to $r \bar{\nabla} r=x \in C$, we have

$$
\operatorname{div}_{C}\left(Y_{1}\right)=\operatorname{div}_{C}(r \bar{\nabla} r)=\frac{1}{2} \triangle_{C} r^{2}+\langle r \bar{\nabla} r, \vec{H}\rangle=\frac{1}{2} \sum \triangle_{C} x_{i}^{2}=\langle x, \vec{H}\rangle+\sum\left|\nabla x_{i}\right|^{2}=n .
$$

On the other hand, for $M=\Sigma$ or $C$,

$$
\operatorname{div}_{M} Y_{2}=\operatorname{div}_{M}\left(r^{-n} Y_{1}\right)=-n r^{-n-1}\left\langle\nabla r, Y_{1}\right\rangle+r^{-n} \operatorname{div}_{M}\left(Y_{1}\right)=n r^{-n}\left(-|\nabla r|^{2}+1\right) .
$$

Note that $|\nabla r| \leq 1$ on $M=\Sigma$ and $|\nabla r| \equiv 1$ on $M=C$. This completes the proof.

Theorem 1. Let $\Sigma$ be a stationary $n$-rectifiable set with boundary $\Gamma$ in $\mathbb{R}^{m}$, an open dense subset of $\Sigma$ being a smooth minimal submanifold. Let $C$ be the cone $p \circledast \Gamma, p \in \mathbb{R}^{m}$. Then

$$
\Theta_{\Sigma}(p) \leq \Theta_{C}(p)
$$

with equality if and only if $\Sigma=C$ and $C$ is star-shaped with respect to $p$.

Proof. Compute the first variation of volume with respect to the (Lipschitz continuous) variation vector field

$$
Y:=r^{1-n} \bar{\nabla} r \quad \text { for } \quad r \geq \varepsilon
$$

and

$$
Y:=\varepsilon^{-n} r \bar{\nabla} r \quad \text { for } r \leq \varepsilon .
$$

Then the first variation of $\Sigma$ with respect to the flow with velocity field $Y$ [Si, p. 80] is

$$
\int_{\Sigma} \operatorname{div}_{\Sigma} Y d V_{\Sigma}
$$

which must equal

$$
\int_{\Gamma}\left\langle Y, \nu_{\Sigma}\right\rangle d V_{\Gamma}
$$

where $\nu_{\Sigma}$ is the outward unit normal vector to $\Gamma$ tangent to $\Sigma$. 
Computing the divergence on smooth subsets of the stationary set $\Sigma$, we find by Lemma 1 (a)

$$
\operatorname{div}_{\Sigma} Y \geq 0 \text { for } r \geq \varepsilon,
$$

with equality at points where $\bar{\nabla} r$ lies in the tangent space, and

$$
\operatorname{div}_{\Sigma} Y=n \varepsilon^{-n} \quad \text { for } \quad r \leq \varepsilon
$$

It follows that for each small $\varepsilon$,

$$
\frac{\operatorname{Vol}\left(\Sigma \cap B_{\varepsilon}(p)\right)}{\left|B_{1}^{n}\right| \varepsilon^{n}} \leq \frac{1}{n\left|B_{1}^{n}\right|} \int_{\Gamma} r^{1-n}\left\langle\bar{\nabla} r, \nu_{\Sigma}\right\rangle d V_{\Gamma},\left|B_{1}^{n}\right|:=\operatorname{Vol}\left(B_{1}^{n}(0)\right) .
$$

Now apply Stokes' theorem to the integral of $\operatorname{div}_{C} Y$ on $C$ :

$$
\int_{C} \operatorname{div}_{C} Y d V_{C}=\int_{\partial C}\left\langle Y, \nu_{C}\right\rangle=\int_{\Gamma}\left\langle Y, \nu_{C}\right\rangle
$$

where $\nu_{C}$ is the outward unit conormal to $\Gamma$ on $C$. Therefore, by Lemma 1 (b)

$$
\frac{\operatorname{Vol}\left(C \cap B_{\varepsilon}(p)\right)}{\left|B_{1}^{n}\right| \varepsilon^{n}}=\frac{1}{n\left|B_{1}^{n}\right|} \int_{\Gamma} r^{1-n}\left\langle\bar{\nabla} r, \nu_{C}\right\rangle d V_{\Gamma}
$$

Note here that

$$
0 \leq\left\langle\bar{\nabla} r, \nu_{C}\right\rangle
$$

and

$$
\left\langle\bar{\nabla} r, \nu_{\Sigma}\right\rangle \leq\left\langle\bar{\nabla} r, \nu_{C}\right\rangle .
$$

Thus, letting $\varepsilon \rightarrow 0$ in inequality (5) and equation (6), we get the desired density estimate. If equality holds, then we must have equality in inequalities (4) and (7), which implies $\Sigma=C$ and $\partial r / \partial \nu \geq 0$.

Definition 1. Let $\pi_{p}$ be the radial projection of $\mathbb{R}^{m} \backslash\{p\}$ onto $\partial B_{1}(p)$, the unit sphere centered at $p \in \mathbb{R}^{m}$. Define the vision angle at $p$ of an $(n-1)$-rectifiable set $\Gamma \subset \mathbb{R}^{m}$ by

$$
\Pi_{p}(\Gamma)=\operatorname{Vol}\left(\pi_{p}(\Gamma)\right)
$$

and the vision angle of $\Gamma$ by

$$
\left.\Pi(\Gamma)=\sup _{p \in \mathbb{R}^{m}} \Pi_{p}(\Gamma)\right) .
$$

Here the volume $\operatorname{Vol}\left(\pi_{p}(\Gamma)\right)$ counts multiplicity.

Clearly we have for any $p \in \mathbb{R}^{m}$ and $C:=p \rightsquigarrow \Gamma$

$$
c_{n-1} \Theta_{C}(p)=\Pi_{p}\left(\Gamma^{n-1}\right) \leq \Pi(\Gamma), \quad c_{n-1}:=\operatorname{Vol}\left(\mathbb{S}^{n-1}\right),
$$

and hence we get the following corollaries to Theorem 1.

Corollary 1. If $\Gamma \subset \mathbb{R}^{m}$ is an $(n-1)$-dimensional compact manifold, then any stationary rectifiable set $\Sigma$ spanning $\Gamma$ satisfies

$$
c_{n-1} \Theta_{\Sigma}(p) \leq \Pi_{p}(\Gamma)
$$


for all $p \in \Sigma$.

Corollary 2. If $\Gamma \subset \mathbb{R}^{m}$ is an $(n-1)$-dimensional compact manifold with $\Pi(\Gamma)<2 c_{n-1}$, then any immersed minimal submanifold $\Sigma$ spanning $\Gamma$ is embedded.

Proof. An immersed submanifold $\Sigma$ with density $\Theta_{\Sigma}(q)<2$ at each point $q \in \mathbb{R}^{m}$ has no self-intersection.

Remark. It may appear inappropriate to view $\Pi(\Gamma)$ as a total curvature. But it has its own merit, as the following example demonstrates. Define an immersed closed $C^{1}$ curve $\gamma \subset \mathbb{R}^{2}$ (the unit square plus four small loops at the corners) by

$$
\gamma=\partial\left([-1,1]^{2}\right) \cup\left\{(x, y):|x|>1,|y|>1,\left[(|x|-1)^{2}+(|y|-1)^{2}\right]^{3 / 2}=\varepsilon(|x|-1)(|y|-1)\right\}
$$

and define a Jordan curve $\Gamma \subset \mathbb{R}^{n}$ to be an embedded $C^{2}$ curve $C^{1}$-close to $\gamma$. Then for small $\varepsilon$,

$$
\int_{\Gamma}|\vec{k}| d s>6 \pi, \quad \text { however, } \quad \Pi(\Gamma) \approx 3 \pi
$$

Hence by Corollary 2 any immersed minimal surface $\Sigma$ spanning $\Gamma$ is embedded since $2 c_{1}=4 \pi$, although the Ekholm-White-Wienholtz theorem [EWW] cannot give the same conclusion.

Let $G_{n}\left(\mathbb{R}^{m}\right)$ denote the Grassmann manifold of $n$-planes through the origin in $\mathbb{R}^{m}$, equipped with the unique $\mathbb{O}(m)$-invariant probability measure, and let $\operatorname{Ave}_{P \in G_{n}\left(\mathbb{R}^{m}\right)}$ be the average over all $P \in G_{n}\left(\mathbb{R}^{m}\right)$. Denote by $\psi_{P}$ the orthogonal projection of $\mathbb{R}^{m}$ onto $P \in G_{n}\left(\mathbb{R}^{m}\right)$.

Lemma 2. Let $\mathbb{S}^{n-1}$ be the unit sphere in $\mathbb{R}^{n} \subset \mathbb{R}^{m}$ centered at the origin $O$ of $\mathbb{R}^{m}$ and let $D$ be a domain in $\mathbb{S}^{n-1}$. Then

$$
\operatorname{Ave}_{P \in G_{n}\left(\mathbb{R}^{m}\right)}\left\{\Theta_{\psi_{P}(O \circledast D)}(O)\right\}=\Theta_{O \circledast D}(O) .
$$

Proof. Assume that $a(D)>0$ is a positive real number such that

$$
\operatorname{Ave}_{P \in G_{n}\left(\mathbb{R}^{m}\right)}\left\{\Theta_{\psi_{P}\left(O \aleph_{D}\right)}(O)\right\}=a(D) \cdot \Theta_{O{ }_{D}}(O) .
$$

Letting $D$ shrink to a point $x \in \mathbb{S}^{n-1}$, one can define a function $a: \mathbb{S}^{n-1} \rightarrow \mathbb{R}$ given by

$$
a(x):=\lim _{D \rightarrow\{x\}} \frac{\operatorname{Ave}_{P \in G_{n}\left(\mathbb{R}^{m}\right)}\left\{\Theta_{\psi_{P}(O \nVdash D)}(O)\right\}}{\Theta_{O \circledast D}(O)} .
$$

Then, by means of a partition of unity by functions of small support, one can see that

$$
a(D)=\frac{\int_{D} a(x) d V_{\mathbb{S}^{n-1}}}{\operatorname{Vol}(D)}
$$


Note here that $\mathbb{O}(n)$ is transitive on $\mathbb{S}^{n-1}$ and that the elements of $\mathbb{O}(n)$ preserve the volume form $d V_{\mathbb{S}^{n-1}}$ on $\mathbb{S}^{n-1}$. Therefore one concludes that for all $x \in \mathbb{S}^{n-1}$,

$$
a(x) \equiv c \quad \text { for a positive constant } c
$$

and hence for any domain $D \subset \mathbb{S}^{n-1}$,

$$
a(D) \equiv c
$$

Therefore it follows from equation (8) that

$$
\operatorname{Ave}_{P \in G_{n}\left(\mathbb{R}^{m}\right)}\left\{\Theta_{\psi_{P}(O \circledast D)}(O)\right\}=c \cdot \Theta_{O \circledast D}(O)
$$

for any domain $D \subset \mathbb{S}^{n-1}$. However, for almost all $P \in G_{n}\left(\mathbb{R}^{m}\right)$,

$$
\Theta_{\psi_{P}\left(O \mathbb{S}^{n-1}\right)}(O)=\Theta_{O \mathbb{S}^{n-1}}(O)=1
$$

Thus $c=1$ in equation (9), which completes the proof.

Theorem 2. Let $\Gamma^{n-1} \subset \mathbb{R}^{m}$ be a compact submanifold. Then

$$
\Pi_{q}\left(\Gamma^{n-1}\right)=\operatorname{Ave}_{P \in G_{n}\left(\mathbb{R}^{m}\right)}\left\{\Pi_{\psi_{P}(q)}\left(\psi_{P}(\Gamma)\right)\right\} .
$$

Proof. The cone $q \circledast \Gamma$ can be thought of as a union of infinitesimal cones $q \circledast \Delta \Gamma_{i}$ and then one can apply Lemma 2 to each $q \circledast \Delta \Gamma_{i}$. Hence

$$
\begin{aligned}
\Pi_{q}(\Gamma) & =c_{n-1} \Theta_{q \nVdash \Gamma}(q) \\
& =c_{n-1} \operatorname{Ave}_{P \in G_{n}\left(\mathbb{R}^{m}\right)}\left\{\Theta_{\psi_{P}(q \circledast \Gamma)}\left(\psi_{P}(q)\right)\right\} \\
& =\operatorname{Ave}_{P \in G_{n}\left(\mathbb{R}^{m}\right)}\left\{\Pi_{\psi_{P}(q)}\left(\psi_{P}(\Gamma)\right)\right\}
\end{aligned}
$$

We shall also require the following generalization of Fáry's theorem to any dimension $n$ and to any codimension $m-n$, which was proved by Langevin and Shifrin ([LS], Proposition $2.15)$ :

Theorem LS. Let $\Gamma^{n-1}$ be a smooth submanifold of $\mathbb{R}^{m}, m \geq n$. Then

$$
\frac{c_{n-1}}{2} \int_{\Gamma} K^{*} d V_{\Gamma}=\operatorname{Ave}_{P \in G_{n}\left(\mathbb{R}^{m}\right)} \int_{\psi_{P}(\Gamma)}\left|G K_{\psi_{P}(\Gamma)}\right| d V_{\psi_{P}(\Gamma)} .
$$

\section{Embeddedness of minimal submanifolds}

It is tempting to propose a higher-dimensional extension of Ekholm-White-Wienholtz's theorem as follows: 
Conjecture. If $q \in \Sigma$, a minimal submanifold of $\mathbb{R}^{m}$ spanning an $(n-1)$-dimensional compact manifold $\Gamma$, then

$$
\Theta_{\Sigma}(q) \leq \frac{1}{2} \int_{\Gamma} K^{*} d V_{\Gamma}
$$

If this were known, one could prove the following as well:

If an $(n-1)$-dimensional compact connected manifold $\Gamma$ satisfies $\int_{\Gamma} K^{*} d V_{\Gamma}<4$, then any immersed minimal submanifold $\Sigma^{n}$ spanning $\Gamma$ is embedded.

Conjecture seems to be hard to prove as yet.

However, if we let $\Gamma_{i}$ be a compact convex hypersurface of an affine $n$-plane $R_{i}^{n} \subset \mathbb{R}^{m}$, $i=1,2$, and define $\Gamma=\Gamma_{1} \cup \Gamma_{2}$, then we may prove Conjecture for this case. Our proof uses the vision angle of $\Gamma$ from a point of $\Sigma$, and averages over projections onto all $n$-dimensional subspaces $P$ of $\mathbb{R}^{m}$. Namely, for $i=1,2$,

$$
\Pi_{\psi_{P}(q)}\left(\psi_{P}\left(\Gamma_{i}\right)\right) \leq c_{n-1}=\int_{\psi_{P}\left(\Gamma_{i}\right)}\left|G K_{\psi_{P}\left(\Gamma_{i}\right)}\right| d V_{\psi_{P}\left(\Gamma_{i}\right)},
$$

since $\psi_{P}\left(\Gamma_{i}\right)$ is a convex hypersurface in $\psi_{P}\left(R_{i}^{n}\right)$. Here, equality holds for all $P$ if and only if $q$ is in $R_{i}^{n}$ and inside $\Gamma_{i}$. Thus we have the following:

Theorem 3. Given two n-planes $R_{1}^{n}, R_{2}^{n}$ in $\mathbb{R}^{m}$, let $\Gamma_{i}$ be a compact convex hypersurface in $R_{i}^{n}, i=1,2$. If $\Gamma=\Gamma_{1} \cup \Gamma_{2}$, then any $n$-dimensional minimal submanifold $\Sigma$ spanning $\Gamma$ is either a union of two flat domains of $R_{i}^{n}$ or is nonflat and has no self intersection.

Proof. We may compute that $\int_{\Gamma} K^{*} d V_{\Gamma}=\sum_{i=1,2} \int_{\Gamma_{i}} K^{*} d V_{\Gamma_{i}}=4$. Thus by inequality (10) and Corollary 1 we have $\Theta_{\Sigma} \leq 2$. If $\Theta_{\Sigma}=2$, inequality (10) and Corollary 1 imply $\Sigma$ is flat. If $\Theta_{\Sigma}<2$ everywhere on $\Sigma$, then $\Sigma$ is nonflat and has no self intersection.

Remark. It should be mentioned that R. Schoen [Sc] proved a theorem which implies a special case of Theorem 3:

If $\Gamma=\Gamma_{1} \cup \Gamma_{2}$ where $\Gamma_{1}, \Gamma_{2}$ are $(n-1)$-spheres in parallel $n$-planes with the line $\ell$ joining their centers being orthogonal to these hyperplanes, then any immersed minimal submanifold $\Sigma^{n}$ spanning $\Gamma$ is a hypersurface of revolution with axis $\ell$. In particular, $\Sigma$ is a catenoid or a pair of plane disks.

\section{References}

[AW] C. B. Allendörfer and A. Weil, The Gauss-Bonnet theorem for Riemannian polyhedra, Trans. Amer. Math. Soc. 53 (1943), 101-129.

[C1] S.-S. Chern, A simple intrinsic proof of the Gauss-Bonnet formula for closed Riemannian manifolds, Annals of Math. 45 (1944), 747-752. 
[C2] S.-S. Chern, On the curvatura integra in a Riemannian manifold, Annals of Math. 46 (1945), 674-684.

[CL] S.-S. Chern and R. K. Lashof, On the total curvature of immersed manifolds, Amer. J. Math. 79 (1957), 306-318.

[EK] J. Eells and N. Kuiper, Manifolds which are like projective planes, Institut des Hautes Etudes Sci. Pub. Maths., 14 (1962), 5-46.

[EWW] T. Ekholm, B. White, and D. Wienholtz, Embeddedness of minimal surfaces with

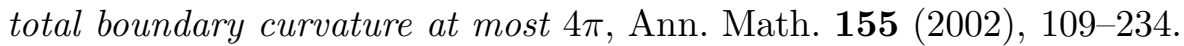

[Fa] I. Fáry, Sur la courbure totale d'une courbe gauche faisant un noeud, Bull. Soc. Math. France 77 (1949), 128-138.

[F1] W. Fenchel, Über Krümmung und Windung geschlossener Raumkurven, Math. Ann. 101 (1929), 238-252.

[F2] W. Fenchel, On total curvature of Riemannian manifolds: I, J. London Math. Soc. 15 (1940), 15-22.

[H] H. Hopf, Über die Curvatura integra geschlossener Hyperflächen, Math. Ann. 95 (1925), $340-367$.

[LS] R. Langevin and T. Shifrin, Polar varieties and integral geometry, Amer. J. Math. 104 (1982), 553-605.

[M] J. Milnor, On the total curvature of knots, Annals of Math. 52(1950), 248-257.

[N] J. C. C. Nitsche, A new uniqueness theorem for minimal surfaces, Arch. Rat. Mech. Anal. 52(1973), 319-329.

[Sc] Richard M. Schoen, Uniqueness, symmetry, and embeddedness of minimal surfaces, J. Differential Geom. 18 (1983), 791-809.

[SS] J. A. Schouten and D. J. Struik, On curvature and invariants of deformation of a $V_{m}$ in $V_{n}$, Proc. Kon. Akad. v. Wetensch. Amsterdam 24 (1922), 146-161.

[Si] L. Simon, Lectures on geometric measure theory, Proc. Centre for Mathematical Analysis, Australian National University, Vol. 3, 1983.

[W] T.J. Willmore, Total curvature in Riemannian geometry, Ellis Horwood Series: Mathematics and its Applications. Ellis Horwood Ltd., Chichester; Halsted Press [John Wiley \& Sons, Inc.], New York, 1982. 
Jaigyoung Choe

Korea Institute for Advanced Study Hoegiro 87, Dongdaemun-gu

Seoul, 130-722

Korea

email: choe@kias.re.kr

fax: +82-2-958-3786

http://newton.kias.re.kr/ choe
Robert Gulliver

School of Mathematics

127 Vincent Hall

University of Minnesota

Minneapolis MN 55414, USA

gulliver@math. umn . edu

+1-612-626-2017

http://www.ima.umn.edu/ gulliver 